\title{
How Does Lighting Direction Affect Shape Perception of Glossy and Matte Surfaces?
}

\author{
Arthur Faisman* and Michael S. Langer ${ }^{\dagger}$ \\ School of Computer Science \\ McGill University, Montreal, Canada
}

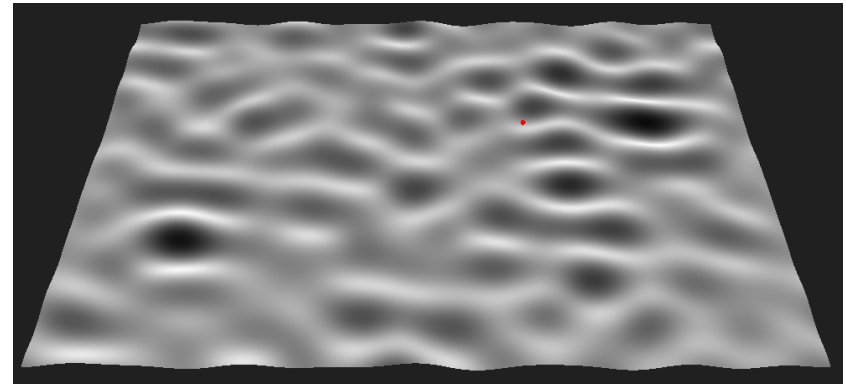

(a) matte

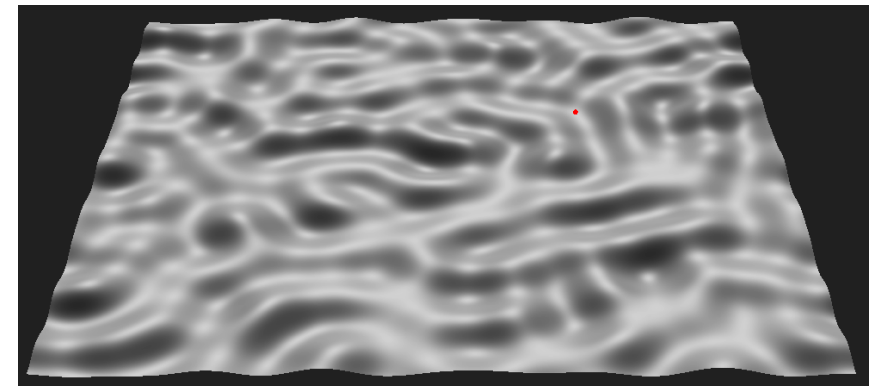

(b) glossy

Figure 1: Sample surfaces at a slant of $\theta=60^{\circ}$ and a light slant of $\phi=120^{\circ}$. Note the more detailed $3 D$ shape percept of the glossy surface, where the specular highlights are located exactly at the tops of the hills and bottoms of the valleys. See Figure 3(a) for definition of $\phi, \theta$.

\section{Abstract}

To visualize the shape of 2D surface data, one often renders it using a simple model with matte or glossy reflectance and a light source at infinity. The parameter choices for this model are typically ad $h o c$, however, and previous studies have provided varying evidence on what this choice should be so that the shape is perceived as accurately as possible. Here we present an experiment that examines local qualitative shape perception on matte and glossy surfaces where we vary both the overall slant of the surface with respect to the viewer and the slant of the distant light source. We find that increasing the slant of the light source to twice that of the surface slant angle improves subjects' perception of qualitative shape of glossy surfaces. Additionally, at these high slant angles the glossy surface percepts are better than those of matte surfaces. We argue that these improvements are due to the positioning of the highlights at the peaks and valleys of the terrain, where they demarcate the surface maxima. We also find that increasing the light slant produces better and/or more consistent shape percepts than the default lighting in commercial visualization software such as Matlab and Mathematica.

CR Categories: I.3.7 [Computer Graphics]: Three-Dimensional Graphics and Realism-Color, shading, shadowing, and texture;

Keywords: shape, shading, highlights, glossy, lighting

\footnotetext{
*e-mail:arthur.faisman@mail.mcgill.ca

†e-mail:langer@cim.mcgill.ca
}

\section{Introduction}

The accuracy of a perceived surface shape can be strongly dependant not only on the shape of the surface but also on its reflectance properties and the scene illuminant. In applications where it's important that the shape be perceived accurately, it is necessary to choose these parameters carefully. Popular commercial data analysis and visualization tools such as MATLAB and Mathematica offer default lighting settings that are independant of the particular surface to be rendered. Other approaches to lighting design optimize some image measure in order to choose from among a set of candidate rendering parameters for rendering full 3D scenes [Shacked and Lischinski 2001; Gumhold 2002; Vázquez 2007].

In this paper, we concentrate on the single surface problem. We investigate how surface reflectance and light direction together affect a viewer's ability to perceive the qualitative shape of the surface. We address two specific questions. The first is, should a rendered terrain surface be made glossy or matte? The second is, at which direction should one place a distant light source? Previous research has tended to address these two questions separately, using either gauge figures to estimate the perceived local surface normals or else having subjects discriminate between two similar shapes.

There have been a variety of studies addressing the first question, i.e. whether subjects can better perceive matte or glossy surfaces. For a fixed lighting direction, Norman et al [1995] and Nefs et al [2006] found no strong differences between matte and glossy conditions, while Todd et al [1997] and Norman et al [2004] found that glossy surface shapes are perceived more accurately. By contrast, in a qualitative shape task, we found that glossy surface shapes were perceived less accurately than matte [Faisman and Langer 2013]. These varied results and the fact that different methods were used in these studies suggest the issue of glossy versus matte might be complex and depend on several factors, including the subject's task (e.g. quantitative versus qualitative shape), the class of surface shapes used (e.g. globally convex blobs versus terrains), and the orientation of the surface and light source relative to the observer.

The second question is how perceived shape varies with the light 

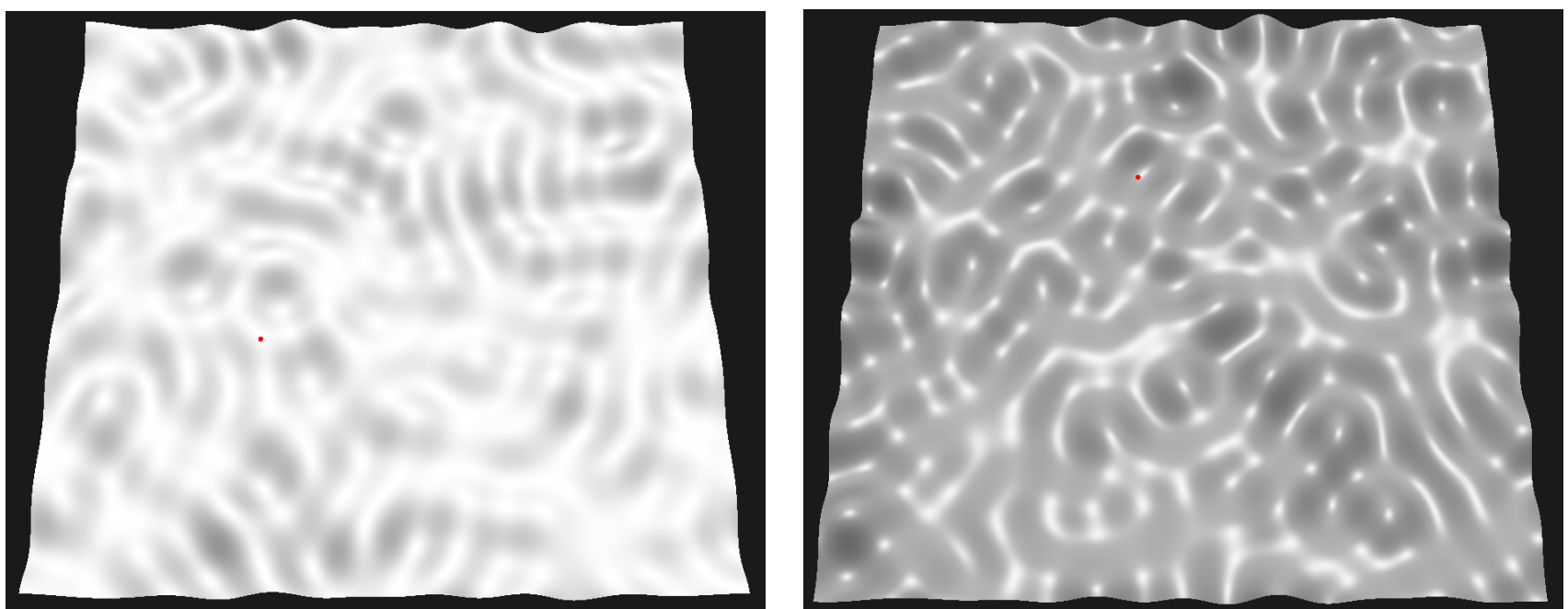

(a)

surface slant $\theta=30^{\circ}$, light slant $\phi=40^{\circ}$

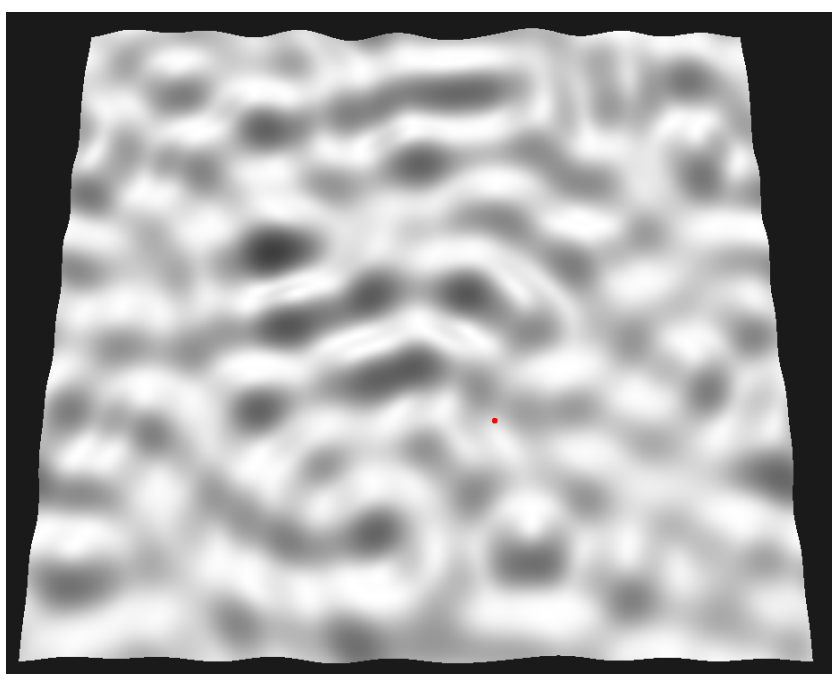

(c)

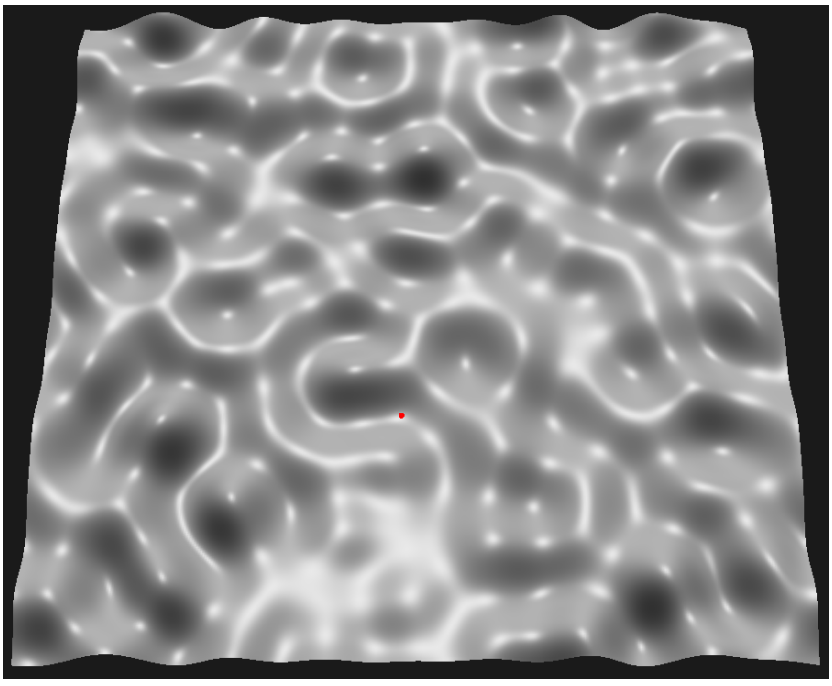

(d)

Figure 2: Sample surfaces of various $\theta, \phi$, and matte (left) and glossy (right). For each surface, a small red dot indicates either a hill or valley. The matte rendering in (a) produces a better shape percept than the glossy surface $(b)$ rendered with the same lighting. On the other hand, the glossy rendering in (d) produces a better shape percept than the corresponding matte rendering (c) because a higher light slant is used. See Figure 3 for definitions of $\phi, \theta$. See experimental results in Figure 5(a). Note that these sample stimuli are best viewed full screen on a monitor, rather than printed paper.

source direction. Koenderink et al [1996] used photographs of glossy mannequins and Christou and Koenderink [1997] used matte rendered ellipsoids to investigate this question. Both found that varying the light source direction caused the perceived shape to deform in the direction of the light. Nefs et al [2005] found similar results but noted that the shape deformations cannot be explained fully by an affine model as had been suggested by Koenderink [1996]. Caniard and Fleming [2007] used glossy surfaces and observed that rotating the lighting direction by 90 degrees in the azimuth strongly altered the subjects' percept of 3D surface shape. Finally, in the study that is closest to our own, O'Shea et al [2008] examined how the elevation of the light source affects the perceived shape. Using globally convex bumpy surfaces, they found that shape was most accurately perceived when the light direction was 20-30 degrees above the line of sight.

The purpose of our experiment is to investigate how the perception of qualitative shape depends on matte versus glossy reflectance for various lighting directions and surface slants. In [Faisman and Langer 2013], we found worse shape percepts for glossy than for matte surfaces for the lighting conditions in Figure 2 (a), (b). The light source slant in this condition is slightly greater than the surface slant, and highlights on the glossy surfaces tend to occur on the front faces of hills rather than at the hilltops (or valley bottoms). We argued that this positioning of the highlights on the front faces may 


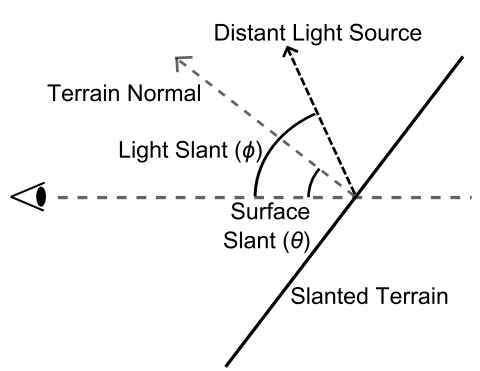

(a)

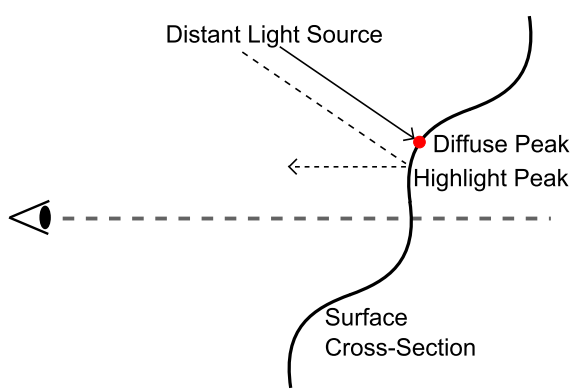

(b) $\phi=\theta$

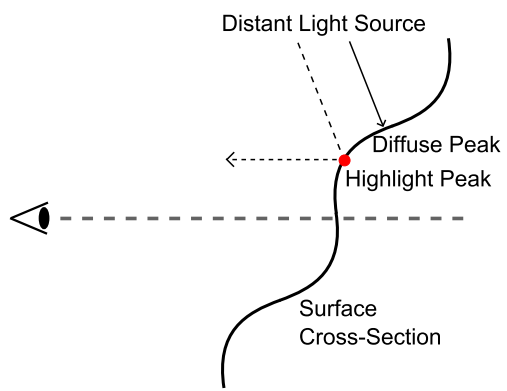

(c) $\phi=2 \theta$

Figure 3: Definitions of the angles used (a) and typical locations of the peak components of the diffuse and highlight lighting components for two configurations of the light slant $(\phi)$ given a constant surface slant $(\theta)$. The case $(b)$ demonstrates $\phi=\theta$ whereas (c) demonstrates $\phi=2 \theta$. Red dot indicates the top of a hill, which is a candidate probe location in the experiment.

interfere with the diffuse shading cue to shape.

In the present study, we consider a range of light source slants including higher light slants. Increasing the light source slant tends to yield specular highlights at or above the hilltops (see Figure 1(b), 2(d)), which is where the diffuse component of the shading peaks as well. (See Figure 3 for illustration, and Section 4 for discussion.) In this sense, the diffuse and specular components give consistent intensity maxima with respect to the underlying shape. Moreover, our subjective impression in viewing the rendered glossy images with greater light slant is that they give a more vivid sense of shape. For these reasons, we hypothesized that greater light source slants would indeed yield better judgments of qualitative shape for glossy surfaces than we found for the light sources used in our previous study (and in other studies).

In addition to varying the slant of the light source, we also systematically vary the slant of the terrain surface to examine if this is an important factor. Finally, we consider two other light source conditions, namely default lighting conditions used in MATLAB and Mathematica, to see if these yield better or worse qualitative shape percepts than the other lighting conditions that we test.

\section{Method}

\subsection{Stimuli}

Similarly to our previous study, [Faisman and Langer 2013], each surface was defined by a $350 \times 350$ mesh terrain rendered using OpenGL. Terrain heights were specified by band-pass filtered noise, from five to nine cycles per surface width. For each surface, a probe point was selected which was either on a convex or concave region (hill or valley). The subject's task was to determine on which type of region a given probe point lay. The probe point was selected randomly from near the center of the surface, with candidate probe points defined as having both principal curvatures above some threshold and of the same sign.

Surfaces were rendered in perspective with the virtual viewer located at $53 \mathrm{~cm}$ from the center. Each surface was rotated back from frontoparallel so that it was upward facing with a slant of either 30, 45, or 60 degrees (see Figures 1, 2, and 4). For each surface slant, a different surface amplitude was chosen. On the one hand, we wanted the surfaces to have large amplitudes so that there would be a large range of surface orientations which would give high luminance contrast. On the other hand, the amplitude needed to be sufficiently small to avoid occluding contours or form shadows for any of the various corresponding lighting conditions (see below).

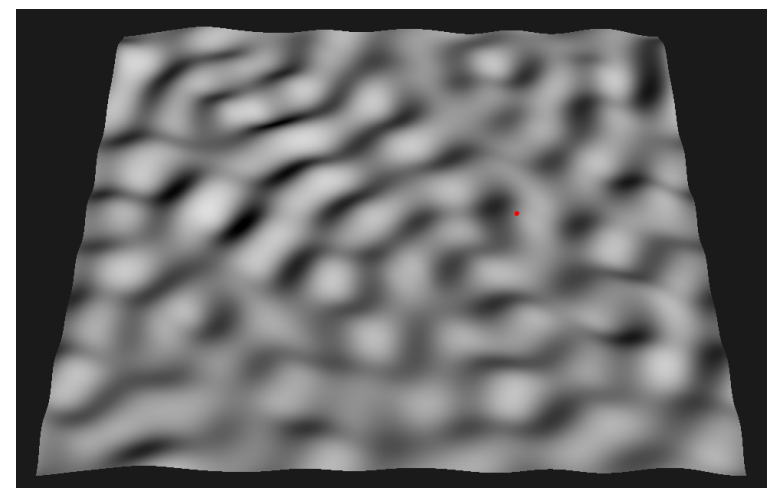

(a) MATLAB matte lighting

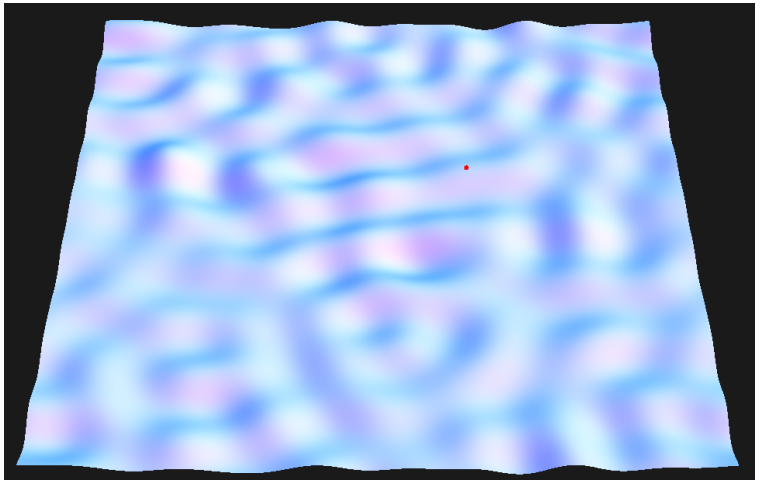

(b) Mathematica lighting

Figure 4: Sample surfaces at a slant of $45^{\circ}$ rendered using the MATLAB and Mathematica default lighting conditions, respectively. See text for details.

These constraints resulted in smaller amplitudes for greater surface slants, with a single different amplitude used for each surface slant.

The surfaces were illuminated using the Phong reflection model [Phong 1975] built into OpenGL. Surfaces were achromatic and two reflectances were used: matte and glossy. The matte surfaces had only a diffuse reflectance and the glossy surfaces were composed of 0.7 diffuse and 0.3 specular. For the specular component, a shininess exponent of 51 was used from the range 0 to 128 allowed by OpenGL. Each surface was rendered against a dark grey background.

Three types of lighting conditions were used. The first used a sin- 


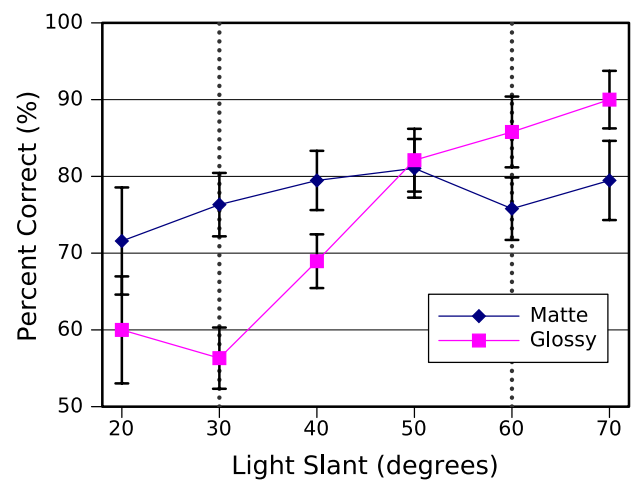

(a) $30^{\circ}$ Surface Slant

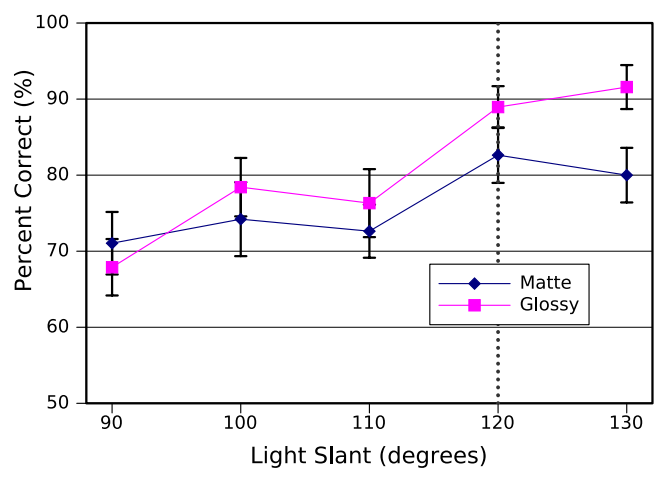

(c) $60^{\circ}$ Surface Slant

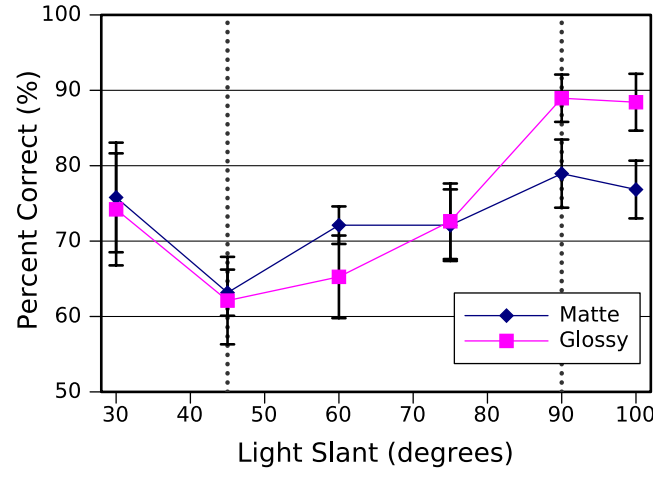

(b) $45^{\circ}$ Surface Slant

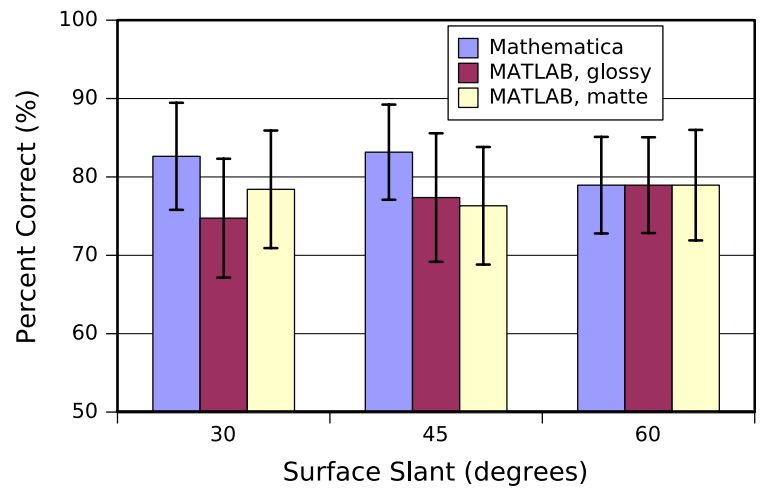

(d)

Figure 5: Percent correct rates for the various surface types and illumination types used. (a) through (c) use a white light source at infinity in direction given by the slant angle $\phi$ (see Fig 3 for schematic). Note that while the light slant $(\phi)$ of $(a)$ and $(b)$ varies from below the surface slant $(\theta)$ to above $2 \theta$, the slants of $(c)$ are limited to a range near $2 \theta$. (d) uses the default lighting styles of two industry standard $3 d$ surface plot rendering programs. Vertical black bars indicate standard error. Vertical dotted lines indicate the light slant which matches the surface slant $(\phi=\theta)$ and the light slant which doubles the surface slant $(\phi=2 \theta)$.

gle directional white light source set at an infinite distance, above and behind the virtual viewer at some angle $\phi$ above the viewing direction (see Figure 3 for schematic). We refer to this angle $\phi$ as the slant of the light source. Note that with this definition, a slant of 90 degrees would be light at the zenith (high noon) and a slant of 180 degrees would be backlighting.

For each of the three surface slants $\theta$, several different light source slants $\phi$ were used. See Figure 5 (a)-(c). For the surfaces slanted at $30^{\circ}$ and $45^{\circ}$, the light source slants included the range $[\theta, 2 \theta]$. For the surfaces slanted at $\theta=60^{\circ}$, the light slants covered a range that was more concentrated near $2 \theta$. Light slants $\phi \approx \theta \approx 60^{\circ}$ were not used in this case because the $\theta=60^{\circ}$ surfaces have only low amplitude hills and valleys and so a light slant $\phi \approx \theta \approx 60^{\circ}$ yielded very low contrast images which were not worth testing.

The second lighting type matched the default lighting direction used by MATLAB, which is a directional light source in the $x z$ plane at 45 degrees azimuth, namely from the direction $(1,0,1)$ where the camera is facing in the $-z$ direction. See Figure 4 . For this lighting condition, both matte and glossy surfaces conditions were used. (MATLAB's default is glossy.) The third lighting type matched the default Mathematica "automatic" lighting condition. This involves three directional diffuse light sources colored red, green, and blue, located at the directions $(1,0,1),(1,1,1)$, and $(0,1,1)$, respectively, with an additional global ambient component.

The stimuli were displayed on a 24 " Apple monitor at $1920 \times 1200$ resolution and a gamma of 2.2 and viewed in a darkened room. We did not use gamma correction. Subjects viewed the stimuli monocularly with an eye patch over the non-dominant eye, and head motion was restrained using a chin rest. The viewing distance was 53 centimeters from the screen which matched the rendering conditions. The resulting stimulus subtended a viewing angle of about $17 \times 13$ degrees.

\subsection{Subjects}

19 subjects participated. Each was 18 years of age or older and had normal or corrected-to-normal vision. Subjects were compensated $\$ 10$ for their time. Results from all subjects are included.

\subsection{Procedure}

In each trial, a new surface was computed and a hill or a valley probe point was chosen. The surface was displayed with a large red frontoparallel disk at the location of the probe point which allowed the subject to make an eye movement to that location. After $350 \mathrm{~ms}$, the large disk was replaced by a small probe point with a diameter of 0.2 degrees visual angle (see Figures 1, 2, and 4 for illustrations).

The subject's task in each trial was to indicate whether the probe point appeared to lie on a hill or in a valley. Each surface was presented for 3.5 seconds total during which the subject had to press one of two buttons on the keyboard indicating hill or valley. For 
more details, see [Faisman and Langer 2013].

\section{Results}

The results are displayed in Figure 5. The first and main trend across all three investigated surface slants $\theta$ is that increasing the light slant $\phi$ beyond $\theta$ increases performance for glossy surfaces. This effect is strongest in the case of $\theta=30^{\circ}$, shown in Figure 5 (a). In general, subjects perform worse (or no better) in the glossy condition than in the matte condition when $\phi<2 \theta$. When $\theta=30^{\circ}$ and $\phi \approx \theta$, subjects performed strictly worse on the glossy rather than matte condition which is consistent with our previous findings [Faisman and Langer 2013]. However, subjects perform strictly better in the glossy condition when $\phi \geq 2 \theta$ which is a new result. For the surface slants $\theta=45^{\circ}, 60^{\circ}$, the strongest single rise in glossy performance appears to be at $\phi=2 \theta$, matching our predictions (see Section 1). This effect is further discussed in Section 4.

One interesting condition is $(\theta, \phi)=\left(45^{\circ}, 30^{\circ}\right)$ which produces a local maximum in performance for that slant. In order to account for this, we note there is little difference between the images in the matte and glossy cases for these low light slants since most of the highlights tend to be reflected away from the observer and hence don't appear in the image. For the light slant $\phi=45^{\circ}$, the diffuse shading component yields a reasonably low contrast image due to the low amplitude of the surface and the fact that the light slant is the same as the surface slant. Therefore, the relatively higher performance at $\phi=30^{\circ}$ may be best viewed not so much as producing a local maximum in performance, but rather as adjacent to the local minimum in performance produced by $\phi=45^{\circ}$. For higher light slants $\phi=60^{\circ}$, the diffuse component starts to come up again, presumably because the light source is slightly oblique to the surface which gives the shading more contrast and serves to increase performance again.

Figure 5 (d) shows the results the MATLAB and Mathematica rendering conditions. Their performance on these conditions was generally better than all but the highest light slants for glossy conditions in Figs. 5 (a)-(c). However, this performance was inconsistent between subjects. There were two subjects who were consistently incorrect (i.e. near $0 \%$ correct) on the MATLAB, Mathematica and $\phi<\theta$ conditions - namely $(\theta, \phi)=\left(30^{\circ}, 20^{\circ}\right),\left(45^{\circ}, 30^{\circ}\right)$, but whose performance was otherwise normal. The unifying feature of these systematically incorrect lighting conditions is that they involve a low light slant, i.e. at $30^{\circ}$ or below ${ }^{1}$. Further studies are needed to determine whether or not these subjects represent a minority of the population that cannot correctly interpret the low slant lighting types used by MATLAB and Mathematica.

\section{Discussion}

Our results showed clearly that increasing the light slant $\phi$ from $\theta$ to $2 \theta$ led to improvements in qualitative shape perception, and that this effect was stronger for glossy rather than matte surfaces. How can these results be explained?

We begin by examining how the diffuse shading and highlights change as the light source slant is increased (see Figures 3,6). For the diffuse component, the intensity peaks at surface points whose normals are parallel to the light direction. More generally, when $\phi<\theta$, the diffuse component peaks appear below the hilltops, when $\phi=\theta$ the diffuse component peaks at the hilltops, and when

\footnotetext{
${ }^{1}$ In fact, these subjects caused the standard errors for all of the conditions with a light source below $\theta$ to be higher than the standard error for all of the conditions that did not involve such a source
}

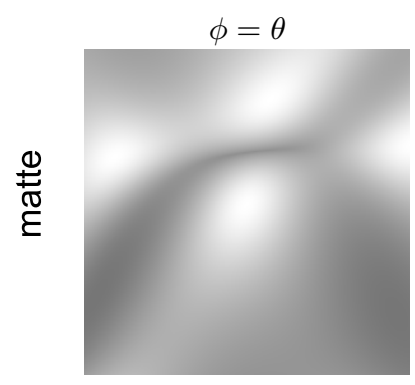

(a)

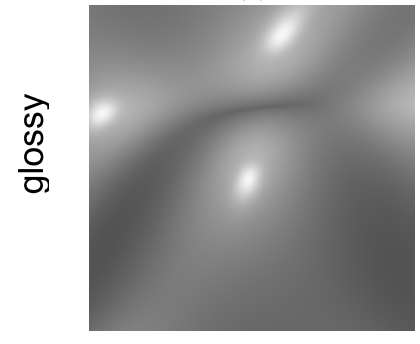

(c)

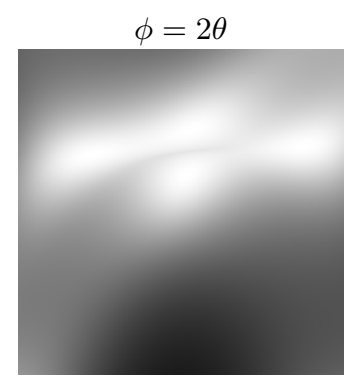

(b)

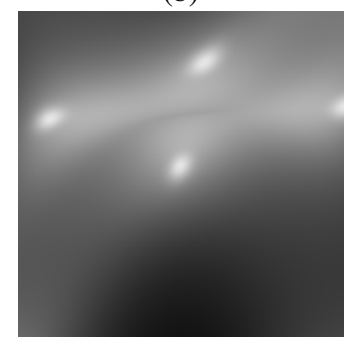

(d)
Figure 6: Sample hills on matte (top) and glossy (bottom) surfaces with a slant $\theta=30^{\circ}$ and light slants $\phi=\theta$ (left) and $\phi=2 \theta$ (right). The images are cropped such that the hilltop is located at the center of each image. When $\phi=\theta$, the peak of the diffuse component is located at the hilltop, and the highlight is located on the front face of the hill, When $\phi=2 \theta$, the highlight is at the hilltop whereas the diffuse shading is located above the hilltop.

$\phi>\theta$ the peaks occur above the hilltops. Note that when the diffuse peak occurs near the hilltop or above it, the local surface is sloped away from the line of sight and the shading become vertically compressed or foreshortened in the image which is arguably an effective qualitative shape cue for the given task [Faisman and Langer 2013].

Highlights behave quite differently. Their intensities peak where the local surface normal is in the direction $\phi / 2$. Thus when $\phi=\theta$, highlights appear below the hilltops; they move to the hilltops when $\phi=2 \theta$, and above the hilltops when $\phi>2 \theta$ (see Figure 3). Note that when $\theta \leq \phi<2 \theta$, highlights appear on the frontally oriented portions of hills. As discussed above, an important diffuse shading cue for these lighting slants is a foreshortening of the intensity distribution above the hilltops. When the highlights occur on the front face of the hills, they can interfere with this diffuse shading cue, resulting in lowered task performance, as for the surface slant $\theta=30^{\circ}$.

By contrast, the presence of a higher-positioned highlight increases task performance. The critical minimum light slant which produced consistently higher performance in the glossy condition seems to be $\phi=2 \theta$. As described above, this is the light slant at which highlights appear exactly at the tops of hills and the bottoms of valleys. Highlights at these locations effectively mark the contours of the surface extrema (recall Figures 1, 2(d)). Further increasing the light slant appears to either benefit or not affect performance. At these high light slants both the highlights and the peaks of the diffuse component are on the oblique part of the surface above the hilltop and are foreshortened, which provides an effective shape cue (see above).

A final factor that may play a role is that at high light slants, the diffuse shading becomes linear ${ }^{2}$ [Pentland 1990]. Linear shading is

\footnotetext{
${ }^{2}$ Linear shading occurs when the surface has a relatively low amplitudes
} 
qualitatively different from the shading that occurs when the light source is above the surface, i.e. $\phi=\theta$. With linear shading, intensity maxima appear where the surface slope is greatest with respect to the underlying terrain, rather than at the hilltops and valley bottoms. One effect is that linear shading tends to produce lower spatial frequencies in the image intensity. For example, in the special case that the surface height is sinusoidal, the spatial frequency of linear shading is the same as that of the surface heights, whereas when $\theta=\phi$ the spatial frequency of the shading is twice that of the surface heights ("frequency doubling"), since the intensity maxima occur at both hills and valleys.

The specular component of glossy surfaces, however, does not give rise to linear shading even at large light slants. One could argue that this is why the glossy shape percepts were better than diffuse ones when $\phi=2 \theta$. This seems plausible when one considers Figures 1 and 2(c,d). The glossy surfaces have higher frequency intensity modulations which better demarcate their hills and valleys than do the diffuse surfaces.

A caveat to the above arguments, however, is that the locations and shapes of both the diffuse and highlight components of the shading can be complex. (See Figure 6, for example.) In general, for example, one can say that there is a bimodal intensity distribution on hilltops corresponding to the peaks of the diffuse and highlight distributions. However, even this simple statement is dependent on many surface properties such as the particular frequencies and amplitudes used to generate the surface, the surface slant, and the highlight exponent supplied to OpenGL. Varying these parameters may in turn change the relative contributions to some of the factors discussed above which affect the surface percept. Further studies are required to elucidate which of these factors affect the percepts more, and under which viewing and rendering conditions.

\section{Conclusion}

We have found that, for glossy surfaces, increasing the light slant so that the highlights are located on the hilltops and valley bottoms tends to improve subjects' ability to judge qualitative shape. For slanted terrains, the light direction slant that achieves this condition is twice the the slant of the terrain. For example, for a glossy surface slanted back at $60^{\circ}$, an optimal light source would be placed at $120^{\circ}$ from the line of sight which amounts to backlighting the terrain. Such a choice probably would not the obvious one to try first, for naive users who are trying to find an effect light source placement when rendering terrains. Indeed commercial software packages such as MATLAB and Mathematica use default lighting conditions that are quite close to the line of sight.

We found that increasing the light slant gave better performance than the MATLAB and Mathematica default lighting directions. However, it would be premature to conclude that this is always the case. A small percentage of our subjects systematically misperceived the surface shapes in the MATLAB and Mathematica conditions, even though they behaved normally in the remaining conditions. This lowered the mean performance in the MATLAB and Mathematica conditions and increased the standard errors. If these (two out of 19) subjects were removed, then performance for these default commercial lighting conditions would have given similar performance to what we obtained by increasing the slant of the light source. Further experiments are needed to clarify how common are such systematic differences in the population.

and when the light source direction is oblique with respect to the overall surface normal. Technically, linear shading arises when the diffuse reflection component $(I=\mathbf{N} \cdot \mathbf{L})$ can be well approximated by a linear function of the surface depth gradient.

\section{Acknowledgments}

This research was supported by a Discovery grant to MSL from the Natural Sciences and Engineering Research Council of Canada (NSERC).

\section{References}

CAniard, F., And Fleming, R. W. 2007. Distortion in 3d shape estimation with changes in illumination. In Proceedings of the 4th symposium on Applied perception in graphics and visualization, ACM, New York, NY, USA, APGV '07, 99-105.

Christou, C. G., And Koenderink, J. J. 1997. Light source dependence in shape from shading. Vision Research 37, 11, 1441-1449.

FAISMAN, A., AND LANGER, M. S. 2013. Qualitative shape from shading, highlights, and mirror reflections. Journal of Vision 13, 5.

GuMHOLD, S. 2002. Maximum entropy light source placement In Visualization, 2002. VIS 2002. IEEE, 275-282.

Koenderink, J. J., van Doorn, A. J., Christou, C., And LAPPIN, I. S. 1996. Perturbation study of shading in pictures. Perception 25, 9, 1009-26.

Nefs, H. T., Koenderink, J. J., And Kappers, A. M. 2005. The influence of illumination direction on the pictorial reliefs of lambertian surfaces. Perception 34, 3, 275-287.

Nefs, H. T., KoenderinK, J. J., And Kappers, A. M. L. 2006. Shape-from-shading for matte and glossy objects. Acta Psychologica 121, 297-316.

Norman, J. F., Todd, J. T., AND Phillips, F. 1995. The perception of surface orientation from multiple sources of optical information. Perception \& Psychophysics 57, 629-636.

Norman, J. F., Todd, J. T., And Orban, G. A. 2004. Perception of three-dimensional shape from specular highlights, deformations of shading, and other types of visual information. Psychological Science 15, 565-570.

O'Shea, J. P., Banks, M. S., And Agrawala, M. 2008. The assumed light direction for perceiving shape from shading. In Proceedings of the 5th symposium on Applied perception in graphics and visualization, ACM, New York, NY, USA, APGV '08, 135-142.

PEntland, A. P. 1990. Linear shape from shading. International Journal of Computer Vision 4, 153-162.

PHONG, B. T. 1975. Illumination for computer generated pictures. Communications of ACM 18, 6, 311-317.

ShACKED, R., AND LischinsKi, D. 2001. Automatic lighting design using a perceptual quality metric. Computer Graphics Forum 20, 3, 215-227.

Todd, J. T., Norman, J. F., Koenderink, J. J., And KaPPERS, A. M. L. 1997. Effects of texture, illumination and surface reflectance on stereoscopic shape perception. Perception 26, $7,807-822$.

VÁZQUEZ, P.-P. 2007. Automatic light source placement for maximum visual information recovery. Computer Graphics Forum $26,2,143-156$. 